\title{
Die Bedeutung imaginativer Geographien im Kampf um „Gorkhaland“
}

\author{
Bishokarma, Miriam
}

\begin{abstract}
Soziale Kämpfe in peripheren Weltregionen werden zunehmend mit raumtheoretischen Kategorien analysiert. Räume unterliegen gesellschaftlichen Konflikten um die Nutzung von Ressourcen, um kulturelle und diskursive Zuschreibungen (etwa „indigener“ Räume und „angestammter“ Territorien), um politische Aneignung und Kontrolle, eng verbunden mit der Ausübung von Herrschaft. PERIPHERIE 126/127 umfasst neben theoretischen Beiträgen empirische Studien zu Kämpfen um Territorien, um konkrete Orte und um die kulturelle Konstruktion von Raum und Identität in unterschiedlichen Weltregionen. Die Artikel spiegeln das breite Spektrum an Fach-disziplinen wider, die sich derzeit mit raumtheoretischen Zugängen zu gesellschaftlichen Konflikten befassen: Soziologie, Politische Geographie, Politische Philosophie, Anthropologie und Regionalwissenschaften.
\end{abstract}

Posted at the Zurich Open Repository and Archive, University of Zurich

ZORA URL: https://doi.org/10.5167/uzh-64633

Journal Article

Published Version

Originally published at:

Bishokarma, Miriam (2012). Die Bedeutung imaginativer Geographien im Kampf um „Gorkhaland“. Peripherie, 32(126/127):296-317. 


\section{Miriam Bishokarma}

\section{Die Bedeutung imaginativer Geographien im Kampf um „Gorkhaland“*}

Keywords: Strategic imaginative geographies, Imaginative geographies, ethno-symbolic resources, ethnoscape, regionalisation, ethno-regional movements, India, Darjeeling, West Bengal

Schlagwörter: Strategische Raumbilder, imaginative Geographien, ethnosymbolische Ressourcen, Ethnoscape, Regionalisierung, ethno-regionale Bewegungen, Indien, Darjeeling, Westbengalen

Seit der Unabhängigkeit im Jahre 1947 befindet sich der indische Nationalstaat in einem Prozess der Reorganisation seiner internen administrativen Grenzen. So hat sich die Zahl der Unionsstaaten seit 1956 von 14 auf 28 verdoppelt, die letzten drei: Jharkhand, Uttarakhand und Chhattisgarh, wurden 2000 gegründet. Trotz dieser weitreichenden Reorganisation, die offiziell auf linguistischen, ethnischen, ökonomischen und ökologischen Kriterien basierte (Bhattacharyya 2001), gibt es noch immer etwa 30 Forderungen meist regional organisierter Gruppen nach eigenen Unionsstaaten. Häufig werden diese Forderungen als Ausdruck der Spannungen zwischen einem pan-indischen Ideal und den Bestrebungen regionaler Gruppen nach mehr Entwicklung, Selbstbestimmung und Schutz ihrer ethnischen und kulturellen Identitäten gedeutet. Diese Forderungen kulminieren in Auseinandersetzungen über Grenzen und die Kontrolle von Territorien und machen diese zu umkämpften Räumen.

Die Forschung zu diesen Bestrebungen fokussiert eher auf Fragen von Identitäts-Politiken oder der Konstruktion von Ethnizität (beispielsweise Baruah 1997; Prakash 2001). Andere Autoren konzentrieren sich auf die Strategien des Staates im Umgang mit diesen Forderungen (Bhattacharyya

* Ich möchte gerne den KritikerInnen sowie der Redaktion der PERIPHERIE für ihre sehr hilfreichen Hinweise sowie meinen BetreuerInnen Ulrike-Müller Böker, Shalini Randeria und Urs Geiser für ihre Unterstützung herzlich danken. Dieser Beitrag wurde im Rahmen des Universitären Forschungsschwerpunkts Europa \& Asien der Universität Zürich, sowie des Swiss National Centre of Competence in Research North-South (NCCR) im Rahmen des Projekts „Research Partnerships for Mitigating Syndromes of Global Change“ verfasst.

PERIPHERIE Nr. 126/127, 32. Jg. 2012, Verlag Westfälisches Dampfboot, Münster, S. 296-317 
2005; Chadda 2002), die umkämpfte Natur Indiens föderaler Ideologie (Adeney 2002; Mawdsley 2002) oder den Zusammenhang mit Wahlpolitiken (Tillin 2011).

In Ergänzung zu diesen Ansätzen verstehe ich in diesem Artikel die Forderungen nach separaten Unionsstaaten vor allem als Auseinandersetzungen über Raum, in denen die offizielle, vom Zentralstaat propagierte Geographie herausgefordert und durch eigene Geographien ersetzt wird. Damit will ich zeigen, dass Elemente des Regionalen - neben Identitäts-, Ethnizitäts-, und Entwicklungsaspekten - eine ebenso bedeutsame Rolle bei Bewegungen für neue Staaten spielen können.

Wenn Territorien und Regionen wichtig sind, so stellt sich die Frage, wie diese Konzepte in der Praxis von den politischen Akteuren in Auseinandersetzungen über neue Unionsstaaten operationalisiert werden und wie diese Raum aneignen. Konkrete Beispiele hierfür sind physisch sichtbare Aktionen durch die Raum markiert und symbolisch angeeignet wird, wie Demonstrationen, Streiks oder Fahnen und Graffitis. Aber wie Edward Said feststellte, geht es beim „Kampf um Geographie“ nicht nur um „Soldaten und Kanonen, sondern auch um Ideen, Formen, Bilder und Vorstellungen“ (Said 1993: 6).

Der vorliegende Artikel verfolgt die These, dass vor allem, ,imaginative Geographien" ein wesentlicher Bestandteil der Strategien sind, mittels derer verschiedene Akteure ihre Ansprüche auf ein umstrittenes Territorium legitimieren und durchsetzen wollen. Diese imaginativen Geographien beziehen sich auf normative Konstruktionen, konkrete Erfindungen und Repräsentationen von geographischem Raum, die, wie ich zeigen werde, über den physischen Raum hinausgehen. Gleichzeitig dienen sie als strategische Mittel der Mobilisierung von UnterstützerInnen, der ideologischen und theoretischen Rahmung des Konflikts und der Stärkung einer regionalen Identität.

Meine These illustriere ich anhand einer Fallstudie. Hierbei konzentriere ich mich auf den Norden des indischen Unionsstaats Westbengalen, speziell auf den Distrikt Darjeeling und dessen angrenzende Gebiete Terai und Dooars, wo die „Gorkhas“ eine Loslösung von Westbengalen in Form eines eigenen Unionsstaates „Gorkhaland“ fordern.

\section{Regionalisierung als politische Strategie}

Entgegen der älteren Annahme, dass Raum und räumliche Strukturen das Handeln von Menschen determinieren, wurde der Zusammenhang zwischen Raum und gesellschaftlicher Praxis seit den 1980er Jahren verstärkt hinterfragt. So werden Kategorien wie „Raum“, „Ort“ (Massey \& Thrift 2003), 
„Scale“ (Marston 2000; Moore 2008), „Grenzen“ (Brunet-Jailly 2005; Van Houtum u.a. 2005) oder „Territorium“ (Delaney 2005; Newman 2010) nicht als gegebene Entitäten verstanden, sondern als durch menschliche Praxis konstruiert und gestaltet. Bereits Lefebvre betonte, dass jede Gesellschaft ihren eigenen Raum produziert (Lefebvre 1991: 53).

Auch „Regionen“ werden nach diesem ,spatial turn“ als durch menschliche Aktivitäten geschaffen begriffen. So beschäftigt sich die „Neue regionale Geographie" mit der Frage des Verhältnisses zwischen der Konstituierung von Regionen und sozialem Leben, Relationen und Identitäten (Paasi 2002: 802). Regionen werden als Aspekte der sozialen Produktion von Raum und als Strategien für politische Mobilisierung begriffen (ebd.: 803). Sie sind somit nicht als feststehende Einheiten, sondern vielmehr als Prozesse und soziale Konstrukte zu verstehen (ebd.: 804).

Auch Bewegungen für neue Staaten in Indien können mit diesen neueren theoretischen Zugängen als Versuche zur Konstruktion neuer Regionen verstanden werden. Sie fordern bestehende Geographien politischer Kontrolle, die Macht innerhalb von territorialen Grenzen festlegen, heraus und versuchen andere politisch-geographische Ordnungen zu produzieren. Regionalismus kann in diesem Kontext als eine „oppositionelle Form des politischen Geographie-Machens“ und als „eine territorial-politisch motivierte Argumentation oder eine soziale Bewegung auf sub-nationaler Ebene" begriffen werden, ,welche die Vertreter des (zentralistischen) nationalstaatlichen Entscheidungszentrum herausfordert" (Werlen 1995: 366). Politische Regionalisierungen sind die Folge von normativen Aktionen (Werlen 2005: 53), durch die Akteure in spezifischen, historisch bedingten Praktiken Räumen bestimmte Bedeutungen zuschreiben (Paasi 2002: 804).

„Regionalisierung“ als aktiver politischer und sozialer Prozess ist unlösbar mit politischen und sozialen Konflikten verknüpft. Unterschiedliche Akteure können stark differierende Vorstellungen über die „,beste“ - oder für sie „nützlichste“ - politisch-geographische Ordnung haben. So beschreibt Anssi Paasi Regionen als Ausdruck eines ,,fortwährenden Kampfes über die Bedeutungen, die mit Raum [und] Repräsentationen [...] verbunden sind“ (Paasi 2002: 805). Diese Kämpfe beinhalten auch Auseinandersetzungen über geographische Vorstellungen, verbunden mit Politiken von Differenzen und Identitäten (MacLeod \& Jones 2001: 679). So fungieren Ideen und Vorstellungen über Regionen und die damit verbundenen Praktiken von Regionalisierungen als konstitutives Element in Identitätspolitiken und als Möglichkeiten, Zugang zu bestimmten Ressourcen zu erlangen (Paasi 2002: 805, 806). Im Folgenden werde ich gezielter auf die Bedeutung von imaginativen Geographien im Regionalisierungsprozess eingehen. 


\section{Imaginative Geographien und strategische Raumbilder}

Die Etablierung einer Region hängt häufig eng mit hegemonialen Narrativen über eine bestimmte räumliche Einheit zusammen, die zu kollektiven sozialen Klassifikationen und Identifikationen führen (Paasi 2002: 805). Solche kollektiven Klassifikationen dienen auch dazu, eine gemeinsame ethnische Identität zu begründen oder zu verstärken. Dabei formen ethno-symbolische Ressourcen wie Erinnerungen, Mythen, Symbole und Werte ein Repertoire, welches Eliten zur Schaffung einer gemeinsamen ethnischen Identität und zur Mobilisierung in nationalistischen Bewegungen nutzen können (Smith 1996b: 590ff). Anthony Smith hat in diesem Zusammenhang drei Grundlagen nationalistischer Bewegungen identifiziert: geteilte Erinnerungen einer reichen Ethno-Geschichte an ein Goldenes Zeitalter, der Glaube an ethnisches Auserwähltsein und die Zugehörigkeit zu einem angestammten „Homeland“ (ebd.). Diese Narrative machen einen bestimmten Raum zu einem unabdingbaren Teil gemeinsamer Erinnerungen, durch die dieser zu der „ewigen“ Heimat der Vorfahren und zum „Eigenen“ wird (Smith 1996b: 589).

Auch imaginative Geographien reflektieren solche Narrative und sind Elemente in der Etablierung von Regionen und der Stärkung eines ethnoregionalen Bewusstseins. Allgemein können imaginative Geographien verstanden werden als „Beschreibungen und diskursive Konstruktionen von Orten, die in einem bestimmten kulturellen Setting gestaltet und neu gestaltet werden" (Radcliffe 1998: 275). Sie sind immer in einen bestimmten soziokulturellen Kontext eingebettet und reflektieren nicht nur die Einstellung, das Weltbild und die Sozialisation von Personen oder Gruppen (Travares \& Brosseau 2006), sondern sind auch aufgeladen mit deren Sorgen und Gefühlen (Radcliffe 1998). Saids Arbeit zu „Orientalismus“ (Said 1978) hat gezeigt, dass solche imaginativen Geographien als Form einer mentalen Raumaneignung zu machtvollen Konstrukten werden können, Ausdruck der Macht, die Welt zu definieren und zu interpretieren (Gregory 1995) - vor allem auch durch eine Verortung des „Eigenen“ und des „Anderen“ (Lossau 2002: 76). In der Verbindung mit Wissen und Macht können imaginative Geographien durch die oben beschriebenen Prozesse der Narrativbildung eine hegemoniale Form annehmen, welche auch die physische Aneignung von Raum legitimiert (Gregory 1995: 463; Ó Tuathail \& Agnew 1992). Als solche sind sie nicht nur Ausdruck einer unbewussten Weltkonstruktion, sondern können als bewusst konstruierte Bilder einen strategischen Charakter erhalten. 
Solche strategisch gestalteten, imaginativen Geographien hat Paul Reuber (1999) „strategische Raumbilder“ genannt. Diese sind selektive Darstellungen und Repräsentationen des beanspruchten Territoriums, die von den Akteuren bewusst eingesetzt werden, um ihre raumbezogenen Zielvorstellungen gegenüber anderen Akteuren durchzusetzen (ebd.). Sie dienen nicht nur der Information und Überzeugung entscheidungsrelevanter Gremien und der Medien, sondern fördern auch die Mobilisierung von UnterstützerInnen und deren Loyalität gegenüber den auf den Raum bezogenen Zielen (ebd.: 34).

Die jeweilige Form der strategischen Raumbilder hängt von verschiedenen Faktoren ab. Zum einen reflektiert sie die räumlichen Zielvorstellungen der Akteure, z.B. die Art und Weise, wie administrative Grenzen gezogen werden sollen, welche Gebiete als zusammengehörig gelten, welche Funktionen ein Raum einnehmen oder welche Form der Regierung von wem über das Gebiet ausgeübt werden soll. Zum anderen ist die Form strategischer Raumbilder Ausdruck des sozio-ökonomischen, politischen und historischen Kontexts, in dem sie entstehen. Für Indien sind insbesondere die historischen Verflechtungen der aktuellen imaginativen Geographien und ihre Zusammenhänge mit dem post-kolonialem Staat und der post-kolonialen Gesellschaft von Bedeutung, wie die Fallstudie zeigen wird.

Reuber hat vier Komponenten von strategischen Raumbildern identifiziert:

- die Festlegung themenrelevanter Kategorien zur Darstellung räumlicher Strukturen (z.B. Bevölkerungsdaten, Siedlungsstruktur, physisches Terrain),

- die einseitige Interpretation dieser Daten,

- die Steigerung der Plausibilität und Richtigkeit des Raumbildes (z.B. durch Bezug auf Gesetze oder Wissenschaft),

- und die gezielte Demontage gegnerischer Raumbilder und die Diskreditierung von Gegnern (Reuber 1999: 330).

Weder die räumlichen Zielvorstellungen noch die strategischen Raumbilder sind statisch, sondern sie passen sich dem Konfliktverlauf an (ebd.: 332). Die vier Komponenten strategischer Raumbilder scheinen allerdings bei der Betrachtung ethno-regionaler Bewegungen nicht ausreichend, denn bei diesen Bewegungen geht es ähnlich wie bei den von Smith beschriebenen nationalen Bewegungen (s.o.) nicht nur um die selektive Präsentation von Daten gegenüber Verwaltungsgremien, sondern vor allem auch um die Stärkung einer gemeinsamen ethnischen Identität. Dies eröffnet Fragen nach der Bedeutung strategischer Raumbilder für eine ethno-regionale Mobilisierung durch Darstellungen geteilter Erinnerungen an ein goldenes Zeitalter, den Glauben an ethnische Auserwählung und die Zugehörigkeit zu einem „Homeland“. Besonders die selektive Darstellung und Interpretation der 
Geschichte des umkämpften Gebietes sind bedeutsam für die Begründung territorialer Ansprüche (Kößler 2007: 405) und zur Mobilisierung von Anhängern (Agnew 2001: 106).

Strategische Raumbilder fördern also eine bestimmte Sichtweise auf die Welt nicht nur in Bezug auf räumliche Daten und Strukturen und auf eine gemeinschaftliche Vergangenheit in einem bestimmten Raum, sondern auch in Bezug auf die Position von Menschen und ihre Beziehungen zum „Anderen“. Bewegungen, die solche Raumbilder propagieren, vermitteln also nicht nur bestimmte Vorstellungen, Ideen und Bedeutungen, sondern produzieren diese aktiv (Snow \& Benford 1988: 188). In der Literatur zu sozialen Bewegungen wird dieser Prozess als „Framing“ (ebd.) bezeichnet. „Frames“ sind: „bewusste strategische Bemühungen von Gruppen [...] zur Herstellung eines gemeinsamen Verständnis der Welt und von ihnen selbst, um kollektives Handeln zu legitimieren und zu motivieren“ (McAdam u.a. 1996: 6). Sie erklären, warum die Welt ist, wie sie ist, und wie sie geändert werden kann und sollte. So werden drei Komponenten des „Framing“ unterschieden: die Identifizierung von Problemen und deren Ursachen (diagnostisches Framing), das Vorschlagen von Lösungen und Lösungswegen für diese Probleme (prognostisches Framing) und die Aufforderung, sich zu engagieren (motivierendes Framing) (Snow \& Benford 1988: 198ff).

Die oben beschriebenen Konzepte sind auf regionalistische Bewegungen in Indien anwendbar und dienen als Basis, auf der ich im Folgenden die Ergebnisse meiner Fallstudie präsentieren werde. Dabei verstehe ich die Forderung nach einem Unionsstaat „Gorkhaland“ als Ausdruck einer regionalen Bewegung, durch die die Akteure eine Neuordnung der administrativen und territorialen Grenzen anstreben. Um diese zu verwirklichen, konstruieren sie imaginative Geographien in Form von strategischen Raumbildern, welche ihre räumlichen Zielvorstellungen legitimieren und Unterstützung mobilisieren sollen. Hierbei sind besonders ethno-symbolische Repertoires bedeutsam, welche die Gefühle der Bevölkerung ansprechen und ein gemeinsames Verständnis des beanspruchten Gebiets als „Homeland“ fördern. Gleichzeitig übernehmen die Raumbilder die Form von „Frames“, mittels derer ein Konsens über Probleme und deren Lösungen propagiert wird.

Auf der anderen Seite versuchen „Gorkhaland“-kritische Gruppen diese Regionalisierungsbestrebungen zu vereiteln und ihre eigenen räumlichen Zielvorstellungen durchzusetzen, wodurch das beanspruchte Territorium zu einem umkämpften Raum wird. Meine Darstellung wird sich besonders auf die Art und Weise der Konstruktion und Dekonstruktion der in diesem Konflikt verwendeten strategischen Raumbilder konzentrieren. 


\section{"Gorkhaland" als imaginative Geographie}

Der Distrikt Darjeeling, vor allem bekannt durch seinen Tee, ist ein umkämpfter Raum, in dem Parteien der „Gorkhas“ eine Separation von Westbengalen und die Gründung eines eigenen Unionsstaats „Gorkhaland“ fordern. Der topographisch vom Vorgebirge des Himalaya geprägte Distrikt, der im Westen an Nepal, im Osten an Bhutan, im Norden an Sikkim und im Süden an Bangladesch grenzt, besitzt eine lange Geschichte sich verschiebender Grenzen und wechselnder Herrscher, und so ist auch die heutige Distriktgrenze das Resultat historischer Prozesse. Diese gilt es kurz zu schildern.

\section{Historischer und politischer Kontext}

Zwischen 1788 und 1815 wurden Teile des damals zum Königreich Sikkim gehörenden Gebietes von dem expandierenden Gorkhakönigreich (heute Nepal) erobert, wohingegen die heutige Kalimpong-Subdivision seit Beginn des 18. Jahrhunderts zu Bhutan gehörte. Nach einem Krieg zwischen der East India Company und Nepal wurden die Grenzen von Nepal durch den Vertrag von Sugauli neu geschrieben und die von Nepal eroberten Areale von den Briten an Sikkim zurückgegeben. 1835 übergab der König von Sikkim die heutigen Darjeeling- und Kurseong-Subdivisionen als „Deed of Grant" an die Briten, die dort eine Hill Station aufbauten. Diese diente neben Erholungsbedürfnissen der Kolonialherren auch militärisch-strategischen Interessen (Chatterji 2007: 84) und wurde zum Element einer „Perlenkette“ solcher Stationen entlang der nördlichen Grenze des Imperiums (ebd.).

Nach einem weiteren Konflikt mit Bhutan wurden schließlich auch die Gebiete des heutigen Kalimpong sowie des Gürtels südlich der bhutanischen Grenze - die so genannten Dooars - 1866 Teil von Darjeeling. Obwohl der Distrikt der Bengal Presidency zugeordnet wurde, besaß er bis zur indischen Unabhängigkeit 1947 einen Sonderstatus als „excluded area“, „backward tract“ oder „scheduled district“ (Samanta 2000: 23), so dass Gesetze, die Landtitel und Steuern betrafen, erst auf besondere Anweisung des Gouverneurs in Kraft traten, um die als „,einfach“ und „tribal“ erachtete Bevölkerung vor Ausbeutung durch andere Gruppen zu schützen. Nach der Unabhängigkeit wurde Darjeeling Teil von Westbengalen, nachdem zunächst die pakistanische Flagge über Darjeeling wehte.

Diese lange Geschichte sich verschiebender Herrschaftsgebiete beeinflusst auch die verschiedenen Raumvorstellungen über Darjeeling. So sehen nationalistische Gruppen in Nepal Darjeeling als Teil eines „Greater Nepal“ an. 
Auf der anderen Seite betrachtet die autochthone Bevölkerung Darjeelings, die Lepchas, „Daar-yjoo-Lyaang“ (wörtlich: „Ort der Götter“) als Teil ihres ursprünglichen Königreichs „Nye Mâyel Renjyong Lyâng“ (wörtlich: „,heiliges verstecktes ewiges Land der Gentlemen") und bezeichnet sich selbst als „Masters of the Land“ (Lyangsong Tamsang, Präsident der Indigenous Lepcha Tribal Association), die nach der Besitznahme der Briten vertrieben worden waren.

Die Kolonialzeit hat nicht nur weitreichende Spuren in der Gestaltung der Landschaft und Architektur Darjeelings hinterlassen (Chatterji 2007), sondern auch eine Umstrukturierung der Bevölkerung verursacht. So wird mit Bezug auf britische Quellen betont, dass vor der Kolonialisierung nur einige wenige Lepchas in dem Gebiet gelebt hätten (ebd.: 92, 117) und dass die Briten den Mangel an Arbeitskräften zum Aufbau der Hill Station durch Förderung der Migration aus Ostnepal und Sikkim ausglichen (Samanta 2000). Diese wurde besonders durch die Gründung von Teegärten, den Bau von Straßen und auch durch die Einrichtung von Rekrutierungszentren für die Britische Armee verstärkt, so dass Nepalesen schnell zur Bevölkerungsmehrheit wurden. Obwohl die Migranten aus unterschiedlichen Sprachund Kastengruppen stammten, entwickelte sich Nepali zur gemeinsamen Sprache; es entstand ein neues Gefühl der Zugehörigkeit als „Gorkha“ über Kasten- und Sprachgrenzen hinweg (ebd.: 23). „Gorkha“ ist jedoch ein unter den Gorkhas selbst umstrittener Terminus zur Bezeichnung von „Nepali sprechenden Indern“ oder „Indern nepalesischen Ursprungs“ (Sinha 2003; Subba 2009).

Erste Forderungen nach Autonomie wurden bereits 1907 von den Anführern der „hill people“ mit Hinweis auf kulturelle Unterschiede zwischen ihnen und den übrigen Gruppen in Bengalen (Subba 1992: 76) formuliert und in den folgenden Jahren wiederholt vorgebracht (Chakrabarty 1988). In den 1980er Jahren formulierte Subash Ghising, der Anführer der Gorkha National Liberation Front (GNLF), schließlich die konkrete Forderung nach „Gorkhaland“, und der bis dahin friedliche Kampf für Autonomie wurde zwischen 1986 und 1988 gewaltreich ausgetragen.

Als Gründe für die Forderung wurden u.a. die Unterdrückung durch Westbengalen und die politische Unsicherheit der Gorkhas mit Bezug auf ihre indische Staatsangehörigkeit genannt (Ghising 1987). Der zweijährige Bürgerkrieg, in dem die Anhänger der GNLF vor allem mit den Anhängern der damals in Westbengalen regierenden Communist Party of India - Marxist (CPI-M) kämpften, wurde 1988 durch die Schaffung einer Autonomiebehörde, des Darjeeling Gorkha Hill Council (DGHC), beendet. Diese 
umfasst drei Hill-Subdivisionen Darjeeling, Kurseong und Kalimong sowie Teile der im Terai ${ }^{1}$ gelegenen Siliguri-Subdivision. 2008 führte jedoch die wachsende Unzufriedenheit der Bevölkerung und das Zusammentreffen mehrerer Ereignisse ${ }^{2}$ zum Sturz des als korrupt, gewalttätig und ineffizient bezeichneten Regimes des DGHC (Benedikter 2009) und seines Anführers Subash Ghising.

Seit 2008 führt eine neue Partei, die Gorkha Janamukti Morcha (GJM, Gorkha Liberation Front - GLF) unter Bimal Gurung den Kampf für „,Gorkhaland" an. Obwohl diese unter dem Motto Gewaltfreiheit, Demokratie und Gandhianism für sich wirbt, geriet sie zunehmend in Kritik, eine Gewaltherrschaft auszuüben und die übrigen Gorkhaparteien ${ }^{3}$ zu marginalisieren. So sind viele Menschen in Darjeeling davon überzeugt, dass die Führungsspitze der GLF 2010 für die grausame Ermordung des Anführers der All India Gorkha League (AIGL), Madan Tamang ${ }^{4}$, der die GLF regelmäßig öffentlich kritisiert hatte, verantwortlich war.

Die GLF betrieb zwischen 2008 und 2011 eine intensive Agitation mit zahlreichen Generalstreiks und Demonstrationen für „Gorkhaland“, unter der neben dem Tourismus auch die Teebranche stark gelitten hat. Im Mai 2011 gewann sie mehr als 80 Prozent bei den Wahlen zur gesetzesgebenden Versammlung Westbengalens in Darjeeling und unterzeichnete im Juli 2011 ein Memorandum mit der neu gewählten westbengalischen Regierung unter Mamata Banerjee (Trinamool Congress). Dieses sieht die Etablierung eines neuen Autonomierats, der Gorkhaland Territorial Administration (GTA), vor. Obwohl Geschäftsleute in Darjeeling das Abkommen mit der Hoffnung auf Frieden und Ruhe begrüßten, kritisierten die übrigen Gorkhaparteien

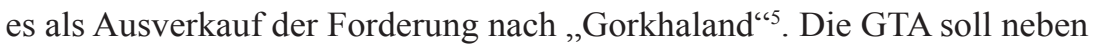

1 Terai bezeichnet das sich im Süden an das Himalayavorgebirge anschließende Flachland.

2 Dies war zum einen Ghisings Plan, die Forderung nach „Gorkhaland“ endgültig aufzugeben, und Darjeeling stattdessen als ,tribal area“ mit weit reichenden Autonomierechten unter dem „Sixth Schedule“ der Verfassung zu versehen - allerdings unter Hoheit des Unionsstaats Westbengalen, und zum anderen die von Bimal Gurung organisierte Unterstützung des aus Darjeeling stammenden Prashant Tamang in dem „Indian Idol“ Talentwettbewerb, durch den große Teile der Bevölkerung mobilisiert wurden. Bimal Gurung gründete daraufhin die GLF, was schließlich zum Sturz der GNLF und zur Vertreibung ihrer AnhängerInnen führte.

3 Dies sind die All India Gorkha League, die Gorkha National Liberation Front, die Communist Party of Revolutionary Marxists sowie die nicht-politische Organisation Bharatiya Gorkha Parisad/Indian Gorkha Council.

4 Madan Tamang, der sich stark gegen die Etablierung eines autonomen Rates für Darjeeling ausgesprochen hatte, wurde am helllichten Tage an einem zentralen Ort in Darjeeling ermordet, als er im Begriff war eine Rede zu halten.

5 Die genaue Beteiligung von Geschäftsleuten am politischen Prozess kann mangels ausreichender Datenlage hier nicht dargestellt werden. Es ist jedoch zu vermuten, dass v.a. die großen Teeplantagenbesitzer ein Interesse an einer schnellen und friedlichen Lösung haben. 
den drei Hill-Subdivisionen auch Teile der Dooars und des Terai umfassen; eine genaue Demarkation des Gebietes steht jedoch noch aus. Die GLF hat wiederholt betont, dass die Gründung der GTA lediglich ein Schritt zu „Gorkhaland“ sei, wohingegen die westbengalische Regierung eine Teilung Darjeelings ablehnt. Weitere Gruppen, die der Gründung von „Gorkhaland“ entgegenstehen, sind u.a. die CPI-M, die Bengalische Gruppe Bangla $O$ Bangla Bhasha Banchao Committee (Save the Bangla Language Committee - BOBBBC) sowie Teile des Akhil Bhraratiya Adivasi Vikash Parisad (All India Adivasi Development Council - ABAVP) und weitere im Terai und in den Dooars etablierte Gruppen.

Soweit die kurze Beschreibung des historischen Kontextes und der Konfliktlinien. Im Folgenden werde ich darstellen, wie „Gorkhaland“ als strategisches Raumbild konstruiert wird, um die Forderungen nach einem eigenen Unionsstaat zu legitimieren. Dabei unterscheide ich primär zwischen „Gorkhaland“ als strategischem Raumbild, mittels dessen eine ethnische Region konstruiert wird, und als Versprechen, durch das nicht nur die lokale Bevölkerung mobilisiert, sondern die Idee auch zu einer nationalen Angelegenheit gemacht werden soll.

Die folgende Beschreibung von „Gorkhaland“ als strategischem Raumbild basiert auf Interviews, die ich im Februar und Juli 2011 mit verschiedenen Vertretern der Gorkhaparteien in Darjeeling geführt habe. Diese werden ergänzt durch die Analyse politischer Reden, von Zeitungsinterviews und des Pamphlets „Why Gorkhaland?“(GLF 2009) der GLF. Die Darstellung der gegnerischen Seite basiert auf einem Interview mit Saman Pathak (CPI-M Darjeeling), den Websites der genannten Gruppen ${ }^{6}$ sowie auf Zeitungsartikeln.

\section{"Gorkhaland" als strategisches Raumbild}

Um die räumliche Zielvorstellung von „Gorkhaland“ zu legitimieren und eine breite Unterstützung in der Bevölkerung zu mobilisieren, nutzen die Akteure der Gorkhaparteien eine Reihe von Strategien, mittels derer die beanspruchten Gebiete von Darjeeling, Terai und Dooars mit Bedeutungen versehen werden. Im Folgenden soll die Konstruktion dieser imaginativen Geographien unter Anwendung der von Reuber und Smith vorgeschlagenen Kategorisierungen dargestellt werden.

6 http://banglabanchao.org; http://dtnm.blogspot.com; http://adivasivikashparishad. blogspot.com. 
Glaube an Auserwähltsein: Der Glaube, als Gruppe auserwählt zu sein, ist laut Smith Teil einer nationalistischen Doktrin, nach der jede Nation eine authentische Identität, eine originale ethnische Kultur, eine Individualität sowie eine besondere Geschichte und Bestimmung besitzen muss, um ihren einzigartigen Beitrag zur Welt aufzuzeigen (Smith 1996a: 453). Die Gorkhaparteien zeigen ihre Einzigartigkeit und Andersheit anhand von zwei Raumbildern auf. Zum einen stellen sie Darjeeling als ,,anderen Ort“ dar, zum anderen als „Zentrum für alle indischen Nepalis“. Die Andersheit entsteht durch die Betonung von ethnischen, kulturellen, physikalischen und historischen Unterschieden zu Westbengalen und den Bengalis. So bezeichnet beispielsweise Alok Thulung (GLF) die Gorkhas als ,distinct race“ (Thulung 2008), die sich durch ihre eigene Sprache, Kultur, Tradition, ihre Art zu Leben und ihre Denkweisen auszeichne. Er nennt auch physikalische Unterschiede und beschreibt Darjeeling als ,topographische Region mit einem komplett anderen Klima“, woraus ,eine natürliche Trennung zwischen den Gorkhas und der Mehrheitsgemeinschaft in Westbengalen" resultiere (ebd.). Auch nutzen die Parteien häufig die Unterscheidung zwischen ,hills“ und ,plains“, um die Andersheit Darjeelings zu betonen.

Ein wichtiger Referenzpunkt für Darjeelings „Andersheit“ und „Einzigartigkeit“ ist auch die koloniale Klassifikation als ,excluded“/,scheduled district" oder als ,backwart tract“, die zeige, dass der Distrikt nie integraler Teil von Westbengalen gewesen sei. Die Argumentation erstreckt sich dabei auch auf die vorkoloniale Zeit. So betont die GLF, dass niemals ein Herrscher aus der bengalischen Ebene über das Gebiet von „Gorkhaland“ geherrscht habe, sondern es sich immer unter der Oberhoheit von Sikkim oder Bhutan befand. Darjeeling sei nur durch verschiedene Abkommen mit ausländischen Mächten an Bengalen angegliedert worden (GLF 2009: 415). Dadurch soll gleichzeitig die Aussage von Gegnern, dass „Gorkhaland“ zu einer weiteren Partition von Westbengalen führe ${ }^{7}$, entkräftet werden.

Als zweites Raumbild zur Stützung des Arguments, ,,anders“ zu sein, dient die Vorstellung von „Darjeeling als Zentrum der indischen Gorkhas“. So nennt R.B. Rai (Communist Party of Revolutionary Marxists - CPRM) Darjeeling das ,soziale, politische, und symbolische Zentrum“ für alle indischen Gorkhas. Es sei nicht nur ein Ort der indischen Freiheitsbewegung, sondern auch Zentrum der Bewegung für die Anerkennung der nepalesischen Sprache in der indischen Verfassung. Zu dieser Rolle trägt nach Ansicht der Parteien auch die Bevölkerungsverteilung bei; so betonen sie die GorkhaMehrheit in den Hügelgebieten Darjeelings, wohingegen „Lepchas, Bhutias

7 http://www.banglabanchao.org/Pages/HomePage.aspx, offizielle Website des BOBBBC, letzter Aufruf: 2. 9. 2011. 
(tibetisch-stämmige Gruppen) und andere“ in der Minderheit seien (GLF 2009: 16).

Geteilte Erinnerungen an ein goldenes Zeitalter: Geteilte Erinnerungen einer Gruppe sind insofern wichtig, als sie zum Gefühl der Zugehörigkeit zu einer Ethnie beitragen (Smith 1996b: 583). Besonders idealisierte Erinnerungen an ein „goldenes Zeitalter“ fördern die Bindung ethnischer Gruppen an einen bestimmten geographischen Raum (ebd.). Auch die Gorkhaparteien bemühen sich, ein „goldenes Zeitalter“ zu beschwören, und stellen dieses ihrer aktuellen Situation gegenüber. Vor allem die GJM zeichnet Darjeeling als ursprünglich reichen Ort des Wohlstands, dessen Einrichtungen aus der Kolonialzeit stammen, und kontrastiert ihn mit der marginalisierten Situation der Gegenwart. Alle Gorkhaparteien sehen in der Vernachlässigung und Ausbeutung durch die Regierung von Westbengalen die Ursachen für den Niedergang. Weiterhin sehen sie eine Bedrohung in den Migranten aus Bangladesch, die sich vor allem im Terai und in den Dooars niederließen und zu demographischen Verschiebungen in diesen Gebieten zum Nachteil der Nepalis beitrugen.

Territorialisierung von Erinnerungen und die Zugehörigkeit zu einem angestammten ,Homeland“: Geteilte Erinnerungen müssen laut Smith an ein spezifisches Territorium gebunden werden, um „,national“ zu werden. Diesen Prozess nennt er „Territorialisierung von Erinnerung“ (Smith 1996a: 453ff). Bestimmte Orte sind Schauplatz für Schlüsselmomente in der Geschichte einer Gemeinschaft, beispielsweise Migrationen. Besondere Bedeutung gewinnen auch die Wirkungsstätten und die Gräber der Vorfahren (Smith 1996b: 588). In den Narrativen bilden diese Räume einen unabdingbaren Teil der geteilten Erinnerungen der Gemeinschaft und werden zum „Homeland“ der Vorfahren, wodurch die territorialen Ansprüche verstärkt werden (Smith 1996a: 454; Smith 1996b: 589). Die Territorien werden zu ,ethnischen Landschaften“ (ethnoscapes) (ebd.; Schetter \& Weissert 2007). Auch Darjeeling besitzt in den Darstellungen der Gorkhaparteien eine lange Geschichte, in der seine Räume durch die Vorfahren angeeignet wurden. So betont H.B. Chettri (GLF), dass das heutige Darjeeling mit seinen Straßen, der Infrastruktur und den Teeplantagen erst durch die Gorkhas geschaffen worden sei:

\footnotetext{
„Alles, was Darjeeling heute ist, ist das Blut und der Schweiß unserer Vorfahren. Es war nicht irgendein Banerjee oder Chatterjee [bengalische Namen], die Darjeeling geschaffen haben.“ (H.B. Chettri, GLF)
} 
Diese Aussage zieht nicht nur eine Grenze zwischen Gorkhas und Bengalis, sondern stellt gleichzeitig eine Verbindung zwischen Körper („Blut und Schweiß") und dem Land her. Darjeeling ist Schauplatz der Einwanderung und der Besiedlung durch die Vorfahren. Die Präsenz von Nepali sprechenden Bewohnern in dem Gebiet wird allerdings nicht nur auf die während der Kolonialzeit stattfindende Einwanderung zurückgeführt, sondern die GLF betont auch unter Hinweis auf ein historisches ,Abkommen von Lo-MenTsong-Tzum “8 aus dem Jahre 1641 die Präsenz der Gorkhas in Darjeeling schon vor der Kolonialzeit, die damit als Autochthone angesehen werden können. Ferner wird die von „Gorkhaland“- Gegnern aus britischen Quellen abgeleitete „Tatsache“ angezweifelt, dass Darjeeling ursprünglich lediglich von 100 Lepchas bewohnt worden sei. Laxman Pradhan (Akhil Bharatiya Gorkha League - ABGL) erklärte sogar die geringe Bevölkerungszahl der Lepcha-Gemeinde damit, dass Lepcha-Frauen häufig britische Offiziere geheiratet hätten, wodurch, ,ihre Produktivität abgenommen hat“. Dies ist bedeutsam, da die Lepchas sich selbst als autochthone Bevölkerungsgruppe in Darjeeling bezeichnen. ${ }^{9}$ Weiterhin betont E.D. Pradhan (Bharatiya Gorkha Parisad/Indian Gorkha Council - BGP), dass viele Gorkhas durch die Neuregelung der Grenze zwischen Nepal und British India zusammen mit ihrem Land in Indien eingegliedert worden seien.

Grenzen der ethno-symbolischen Mobilisierung? Die Forderung nach „Gorkhaland" bezieht sich nicht nur auf den Distrikt Darjeeling, sondern auch auf die angrenzenden Gebiete der Dooars und des Terai, deren Bevölkerung neben Nepalesen v.a. aus Adivasi besteht. Die Gorkhaparteien möchten die Gebiete mit einer nepalesischen Mehrheit dem neuen „Gorkhaland“ eingliedern. Während die Nepali sprechende Bevölkerung diese Forderung überwiegend unterstützt, stehen ihr die übrigen Bevölkerungsgruppen kritisch oder ablehnend gegenüber. Als Hauptargumente für die ,Zusammengehörigkeit der Region Darjeeling, Dooars, Terai“ 'betonen die Gorkhaparteien, bezogen auf kulturelle, soziale und sprachliche Aspekte, Gemeinsamkeiten mit den anderen Gruppen, wie inter-ethnische Beziehungen z.B. Hochzeiten (GLF 2009: 6) oder die gemeinsame Opferrolle unter der ,Tyrannei der Mehrheit“ (ebd.: 10) in Westbengalen. Auch wird hervorgehoben, dass die Gebiete Mal, Matheli und Samsing (in den Dooars) bis 1976 für die Wahl zur Lok

8 Dieses Abkommen umfasste die Gebiete von Sikkim und Darjeeling. Angeblich sind nepalesische Gruppen (Limus, Subbas, Magars) seine Unterzeichner.

9 Die Lepchas übertragen ihre Ansprüche nicht in territoriale Forderungen, haben jedoch im September 2011 nach Demonstrationen die Zusage zu einem „Lepcha Development Council" von der westbengalischen Regierung erreicht. 
Sabha ${ }^{10}$ Teile des Wahlkreises von Darjeeling gewesen seien. Der deutlichste Versuch, die Dooars und Darjeeling als zusammengehörige Region zu präsentieren, war wohl die kurzzeitige Umbenennung von „Gorkhaland“ in „Gorkha Adivasi Pradesh“ durch die GLF ${ }^{11}$, eine Idee, die sich vermutlich auch deshalb nicht durchgesetzt hat, weil die Nicht-Nepali-Gruppen sich in der Argumentation der Partei kaum wiederfinden konnten.

Darjeeling als Ethnoscape: Die Darstellung raumbezogener und historischer Aspekte Darjeelings resultiert in verschiedenen Raumbildern - Darjeeling als Ort der Vorfahren, als ehemals „reicher“ Ort, als ,,anderer“ Ort, als „Zentrum“, als zusammengehörig mit Dooars und Terai-, die nicht nur die Forderung nach Separation von Westbengalen legitimieren sollen, sondern auch mittels Bezugnahme zu ethnischen Symbolen eine starke mobilisierende Wirkung erlangen. So geht die Argumentation der Gorkhaparteien über eine rein rationale Darstellung raumbezogener Gegebenheiten hinaus. Vielmehr verknüpfen die imaginativen Geographien Geschichten von Besiedlung, Migration und menschlicher Aneignung mit dem physischen Raum von Darjeeling/Dooars/Terai. In diesen Narrativen sind die imaginierten Verbindungen zwischen der kolonialen Vergangenheit und der Gegenwart immer präsent. Dadurch wird das geschaffen, was Paasi eine „regionale Identität“ bezeichnet hat, also eine kollektive Identifizierung der Menschen mit ihrer Region. Diese erlangt nicht nur Gewicht als Komponente von Territorialität und als signifikantes Element in der Konstruktion von Regionen als bedeutungsvolle sozio-politische Räume (Paasi 2001: 138), sondern auch als Element von Ethnizität. Darjeeling wird dadurch zu einem Raum der „Gorkhas“, zu einem „,ethnoscape“ (Smith 1996a: 453).

Ein weiterer Blick zeigt jedoch, dass das strategische Raumbild „Gorkhaland" über die Konstruktion von Narrativen durch die Gorkhaparteien hinausgeht. Das Bild von „Gorkhaland“ dient auch als „Frame“, der „Gorkhaland" als Lösung von Problemen präsentiert. Ich nenne diesen Aspekt des Raumbildes „Gorkhaland als Versprechen“.

\section{"Gorkhaland" als Versprechen}

Obwohl dieser Beitrag nicht anstrebt, die Ursachen für die Forderungen nach „Gorkhaland“ zu erforschen, so ist für ihr Verständnis wichtig, wie die Gorkhaparteien sie mit Bezug zu sozio-ökonomischen, politischen

10 „Volksversammlung“, das Unterhaus des indischen Parlamentes.

11 „GJM now for Gorkha-Adivasi Pradesh“. In: The Hindu vom 1. 6. 2010, http://www. thehindu.com/news/article442973.ece, letzter Aufruf: 2. 10. 2011. 
und kulturellen Faktoren begründen und welche Bedeutung imaginative Geographien hierbei spielen. Ein Großteil der Unterstützerbasis für die Bewegung stammt aus den Teeplantagen, die seit Jahrzehnten mit sinkenden Produktionszahlen, niedrigem Lohnniveau und Schließungen zu kämpfen haben. Es liegt daher nahe, dass die Forderung nach „Gorkhaland“ auch im Kontext von Entwicklungsbestrebungen formuliert wird. So beklagen die Gorkhaparteien nicht nur einen Mangel an guter Infrastruktur, an Arbeitsmöglichkeiten und an Partizipation in politischen Entscheidungsprozessen, sondern auch die ,neokoloniale Herrschaft“ der westbengalischen Regierung, welche die Gorkhas zu marginalisierten und ausgebeuteten Bürger zweiter Klasse mache. Neben diesen sozio-ökonomischen Aspekten nennen jedoch alle Parteien einstimmig die „Identitätskrise“ der Gorkhas als Hauptgrund für die Forderung nach „Gorkhaland“. Mit dem Begriff „Identitätskrise“ bezeichnen sie ihre wahrgenommene Stigmatisierung als nepalesische StaatsbürgerInnen und die Nicht-Anerkennung als indische StaatsbürgerInnen.-Die Ursachen für diese fehlerhafte Wahrnehmung sehen sie vor allem in körperlichen, sprachlichen und kulturellen Ähnlichkeiten mit Nepalesen sowie in der offenen Grenze zu Nepal. Diese „Identitätskrise“ ist eng verbunden mit einem Gefühl der Unsicherheit und der Angst vor Vertreibung und ethnischer Gewalt. Der Darstellung Darjeelings als verarmter, von der Regierung unterdrückter und vernachlässigter Region und der Gorkhas als Opfer einer rassistischen Politik, die sie als nicht-indische Nepalesen stigmatisiert, wird die Vision von „Gorkhaland“ gegenübergestellt. Dabei steht die Vorstellung, dass eine Separation von Westbengalen in der Form einer territorialen Grenze die „Identitätskrise“ lösen könne, im Vordergrund, „denn die Grenze ist das, was Identität kreiert“ (H.B. Chhetri), oder anders ausgedrückt: „Gebt uns 'Gorkhaland' [...], und automatisch bekommen wir eine Adresse“ (E.D. Pradhan, BGP) ${ }^{12}$. Diese räumliche Logik bezieht sich nicht nur auf Westbengalen, sondern auch auf Nepal, denn die Schaffung von „Gorkhaland“ wird auch als Trennung von Nepal gewertet. Gleichzeitig stellen die Parteien die Gründung von „Gorkhaland“ als Weg zur stärkeren Verbindung mit der Indischen Union dar, denn nur durch einen eigenen Unionsstaat könne Partizipation in nationalen Entscheidungsprozessen gewährt werden (H.B. Chhetri, GLF).

In diesem Zusammenhang wird „Gorkhaland“ auch als nationale Angelegenheit präsentiert, denn in Indien obliegt die Entscheidung darüber, ob ein

12 Hierbei verweisen die Akteure auch auf andere Gruppen in Indien, wie die Tamilen oder Punjabi, deren Zugehörigkeit zum indischen Staat - trotz der Nachbarschaft zu Sri Lanka oder Pakistan - nicht angezweifelt würde, da sie eigene Unionsstaaten (Tamil Nadu, Punjab) besitzen. 
neuer Staat gegründet werden soll, der Zentralregierung. Dieses „Scaling“ (Jones \& Fowler 2007; Moore 2008) erfolgt durch eine Verknüpfung der Forderung mit dem nationalen Sicherheitsdiskurs. So betonen die Gorkhaparteien, dass die geostrategisch sensible Region rund um den „Chicken's Neck“ (Ramachandran 2011) mit Grenzen zu Nepal, Bangladesch und Bhutan, und (über Sikkim) China, die über einen schmalen Korridor den gesamten Nordosten mit dem indischen Hauptland verbindet, nur durch „Gorkhaland“ geschützt und stabilisiert werden könne. „Gorkhaland“ sei eine „Festung“ (H.B. Chhetri, GLF) und ein „Puffer-Staat, bewohnt von loyalen Gorkhas“ (GLF 2009: 12, 13), durch die Sicherheit gegen ausländische Infiltration und Terrorismus ${ }^{13}$ gewährleistet werde. Die Verbindung zu geopolitischen Interessen der indischen Regierung erfolgt so unter Rückgriff auf die Zuschreibung der Gorkhas als loyale und mutige Kämpfer, wie sie von den britischen Kolonialherren in der ,,martial race“-Theorie propagiert wurde (Caplan 1995). Auch durch Bezug zu Artikel 3 der indischen Verfassung, der die Reorganisation von Staaten vorsieht, untermauern die Gorkhaparteien die Rechtmäßigkeit ihrer Forderung und stellen sich als Gruppe innerhalb der indischen Union dar, welche die nationale Integrität nicht in Frage stellt.

Zusammenfassend erlangt „Gorkhaland“ nicht nur die Bedeutung eines eigenen separaten Staats auf einem bestimmten Territorium, sondern erscheint als Garant für Gerechtigkeit, Gleichheit, Partizipation, Anerkennung der indischen Identität, für Autonomie und Demokratie sowie für nationale Sicherheit und Schutz vor ausländischer Bedrohung präsentiert. „Gorkhaland“" verspricht somit eine Lösung für die genannten Probleme von Marginalisierung, Mangel an Entwicklung und Unsicherheit, und übernimmt die Funktion von diagnostischen und prognostischen „Frames“. Gleichzeitig verknüpft das Raumbild die Vorstellungen von einem „Homeland“ und einer gemeinsamen Bestimmung der Gruppe durch den Glauben daran, dass Selbstrealisierung nur auf dem ,eigenen Boden“ möglich sei und dass das Land von Unterdrückern befreit werden müsse (Smith 1996b: 590). In diesem Sinne wird „Gorkhaland“ zu einem Versprechen einer besseren Welt.

\section{Die Dekonstruktion von "Gorkhaland"}

Während die Gorkhaparteien das strategische Raumbild von „Gorkhaland“ propagieren, gibt es zahlreiche Gegner. Dies sind neben der bis 2011 regierenden CPI-M auch im Terai und Dooars organisierte Gruppen, wie das

13 „PC to Morcha: Go for More Talks - Hill Delegation Offers Chicken Neck Stability“. In: The Telegraph vom 30. 12. 2008, http:/www.telegraphindia.com/archives/archive.html, letzter Aufruf: 1. 11. 2011. 
BOBBBC, der ABAVP oder Dooars Terai Nagarik Manch (DTNM), die hier exemplarisch vorgestellt werden. Auch die neu gewählte Regierung von Mamata Banerjee und dem Trinamool Congress (TMC) ist gegen „Gorkhaland“. Obwohl diese Gruppen unterschiedliche Ziele verfolgen - von der Steigerung der Macht der Unionsstaaten (CPI-M) über den Schutz und die Förderung von Bengalis (BOBBBC) bis hin zur einer eigenen Autonomiebehörde für die Dooars -, ähneln sie sich in ihren Argumentationen.

Die im Terai/Dooars verankerten Gruppen (BOBBBC, DTNM, ABAVP) verfolgen vor allem eine Strategie der Diskreditierung der Gorkhaparteien und ihrer Forderungen. So stellen sie „Gorkhaland“ als Bedrohung für die nationale Integrität dar. Die Gründung von „Gorkhaland“ führe zu einer „Balkanisierung Indiens“14 und sei eine Verschwörung für ein „Greater Nepal", das manche nationalistische Gruppen in Nepal fordern (Unified Nepal Nationalist Front 2011). Die TDNM sehen in „Gorkhaland“ die Gefahr einer Vertreibung von Indern aus Darjeeling. Darjeeling wird als integraler Teil von Westbengalen dargestellt, der gewaltsam und illegal von Nepalesen überrannt und den Bengalen und Lepchas als den „wahren Söhnen der Erde“" gestohlen wurde. Die Gegner untermauern diese Argumentation durch Bezug auf Bevölkerungsstatistiken, Wachstumsraten und auf koloniale britische Quellen. Dabei nehmen sie jedoch keinen Bezug auf Darjeelings historischen administrativen Sonderstatus. Dies zeigt, wie auch hier geschichtliche Daten selektiv zum eigenen Nutzen dargestellt werden.

Die Argumentation der CPI-M ist im weniger radikal. Vielmehr betont Saman Pathak (CPI-M Darjeeling), dass Forderungen nach Autonomie und Selbstbestimmung durch das ,Staat-im-Staat“-Konzept erfüllt werden könnten, also durch die Gründung autonomer Räte unter der Hoheit der jeweiligen Staaten. Nur starke Staaten könnten die Macht des Zentrums eingrenzen, kleinere Staaten würden in ihrer Entwicklung behindert und hätten kaum Macht. Statt der Zugehörigkeit zu einer ethnischen Gruppe müsse das Klassenbewusstsein gestärkt werden.

Auch die neu gewählte Regierung Westbengalens unter Mamata Banerjee ist gegen eine Abspaltung Darjeelings. Obwohl die Gorkhaparteien ihre Unterschiede zu den Bengalis betonen und einen ethnische und kulturellen Trennstrich ziehen, hat Banerjee wiederholt mit Bezug auf Darjeeling von ihren „Brüdern und Schwestern“ gesprochen und Darjeeling als ihr „Darling“ und „Baby“ beschreiben. ${ }^{15}$ Dies wurde auch in der Zusage von großzügiger

14 http://www.banglabanchao.org/Pages/HomePage.aspx. Offizielle Website des BOBBBC, letzter Aufruf: 2. 9. 2011.

15 „Unity on Lips, CM Serenades Dar(jee)ling“. In: The Telegraph vom 12. 10. 2011, http://www. telegraphindia.com/1111012/jsp/frontpage/story_14614119.jsp, letzter Aufruf: 14. 10. 2011. 
finanzieller Unterstützung deutlich. Die Vision von „Gorkhaland“ versucht sie dabei durch das Versprechen, aus Darjeeling eine „Schweiz“ zu machen, zu ersetzen - mit der Bedingung, dass die „Schweiz“ innerhalb von Westbengalen bleiben müsse. Ob diese Strategie Erfolg haben wird, ist jedoch eine offene Frage, denn die GLF hat bereits betont, dass sie die Forderung nach „Gorkhaland“ nicht aufgeben wird.

\section{Zusammenfassung - Die Macht von imaginativen Geographien?}

Das Ziel dieses Beitrags war es zu zeigen, dass bei Bewegungen für neue Staaten in Indien regionalistische Aspekte eine ebenso wichtige Rolle spielen wie vorgestellte Identitäten und ethnische Konstrukte. Dabei wurde die These aufgestellt, dass besonders die Konstruktion von imaginativen Geographien in Form von strategischen Raumbildern eine wesentliche Strategie zur Legitimierung und Mobilisierung darstellt. So formulieren die Akteure ihre Forderungen bewusst mit Referenzen zu raumbezogenen Daten wie Geschichte, Bevölkerungszahlen oder Migrationsbewegungen. Der Bezug zum früheren administrativen Sonderstatus des Distrikts und zum kolonialen Wohlstand reflektiert gleichzeitig die Verflechtung der Forderung der Gorkhaparteien mit der kolonialen Vergangenheit. Das umstrittene Territorium wird mit einer Geschichte von Aneignung und Besiedlung verknüpft und mit emotionalen Aspekten versehen, die insgesamt die Grenzziehungen um den umkämpften Raum-gegenüber der Regierung, den Medien und Entscheidungsgremien rechtfertigen und eine regionale Identität formen sollen.

Wie die Fallstudie zeigt, sind die Raumbilder geprägt von Referenzen zu ethno-symbolischen Ressourcen. Sie propagieren die Vorstellung eines ethnischen Auserwähltseins, eines ,,goldenen Zeitalters“, sowie der Zugehörigkeit zu einem ursprünglichen „Homeland“. In dieser Verbindung erhalten die Raumbilder ihr volles mobilisierendes Potential, da sie Gefühle der Bevölkerung direkt ansprechen und ausnutzen (Smith 1996a: 458).

Dabei zeigt sich in der Konstruktion des Bildes „Gorkhaland“ eine räumliche Containerlogik, in welcher ein Glaube an eine Schaffung von Identität durch territorial-administrative Grenzen, also an eine Isomorphie zwischen Raum und Ethnie dominant ist. „Gorkhaland“ wird in dieser Argumentation zu einem „ethnoscape“ (Schetter \& Weissert 2007; Smith 1996a), in dem das Bewusstsein einer ethnischen Zugehörigkeit als „Gorkha“ an das Vorhandensein eines gemeinsamen physischen Raumes geknüpft ist. Gleichzeitig entfaltet die Vision von „Gorkhaland“ ihre volle Macht durch die narrativen Verknüpfungen von imaginierten Räumen mit den physischen Räumen von 
Darjeeling/Dooars/Terai. Die geographische Imagination von „Gorkhaland“ beschreibt die Welt nicht nur, wie sie anscheinend ist, sondern auch wie sie sein sollte. Kämpfe um BürgerInnenrechte, Identität, Entwicklung und Anerkennung von Identität werden dabei als Kämpfe um das Territorium ausgetragen. „Gorkhaland“ beinhaltet das Versprechen einer neuen, besseren, gerechteren Welt. Dadurch erhält es Elemente einer Utopie, eines ,ortlosen Raums“, eines imaginierten, ,fundamentalen unwirklichen Raum[s]“" (Foucault \& Miskowiec 1986: 24), der noch nicht Realität geworden ist.

Doch obwohl sowohl die Bewegung für „Gorkhaland“ als auch andere Autonomiebewegungen eine innere Reorganisation Indiens fordern, so werden diese Forderungen doch in der räumlichen Logik des Nationalstaats formuliert. Diese wird besonders durch die Verbindung mit ethno-symbolischen Ressourcen deutlich, welche nicht nur eine mobilisierende, sondern in der Logik nationalen „Mainstream“-Denkens auch eine legitimierende Funktion haben. So wird keineswegs der Glaube an die Zusammengehörigkeit von Kultur und Raum oder die generelle Vorstellung einer territorial organisierten Welt herausgefordert. Statt die Grundfesten des Staates zu erschüttern und zu hinterfragen, werden diese gerade durch Forderungen nach Autonomie in Form von Unionsstaaten reproduziert und unterstützt. Territorien bleiben damit im Zentrum von oftmals gewaltsamen Konflikten, da mit der Vorstellung eines „eigenen Raums“ auch die Vorstellung eines besseren Lebens verknüpft wird. Jedoch stellt sich besonders für die Gruppe der Nepali sprechenden Inder, die sich über den gesamten indischen Subkontinent verteilt, die Frage nach der Sinnhaftigkeit einer territorialen Lösung ihrer Probleme. So bietet vielleicht der Vorschlag des CPI-M-Mitglieds Saman Pathak, den indischen NepalisprecherInnen den Status einer linguistischen Minderheitengruppe zu verleihen, was bestimmte Quoten innerhalb der indischen Gesellschaft garantieren würde, die Möglichkeit einer nichtterritorialen Lösung. Doch grade die Vorstellung, dass die Gründung eines eigenen Unionsstaats die Probleme lösen könne, wird durch die Konstruktion von „Gorkhaland“ als imaginativer Geographie festgeschrieben. Die zum Teil gewaltsame Marginalisierung anderer Gorkhaparteien durch die GLF wirft auch die Frage auf, wie Akteure die Vision von „Gorkhaland“ nutzen, um Herrschaft und Kontrolle über Darjeeling zu legitimieren und möglicherweise andere Motive zu verbergen. 


\section{Literatur}

Adeney, Katharine (2002): „Constitutional Centring: Nation Formation and Consociational Federalism in India and Pakistan“. In: Commonwealth \& Comparative Politics, Bd. 40, Nr. 3, S. 8-33.

Agnew, John (2001): „Regions in Revolt“. In: Progress in Human Geography, Bd. 25, Nr. 1, S. 103-110.

Baruah, Sanjib (1997): „Politics of Subnationalism: Society Versus State in Assam“. In: Partha Chatterjee (Hg.): State and politics in India. New Delhi, S. 496-520.

Benedikter, Thomas (2009): „Gorkhaland: Autonomy is no Longer the Issue“. In: Benedikter, Thomas (Hg.): Solving Ethnic Conflict through Self-Government. A Short Guide to Autonomy in South Asia and Europe. Bozen, S. 104-111.

Bhattacharyya, Harihar (2001): „India Creates Three New States“. In: Federations, Bd. 1, Nr. 1., S. ?.

Bhattacharyya, Harihar (2005): Federalism and regionalism in India. Institutional strategies and political accommodation of identity. Heidelberg Papers in South Asian and Comparative Politics. Working Paper, Nr. 27, Heidelberg.

Brunet-Jailly, Emmanuel (2005): ,Theorizing Borders. An Interdisciplinary Perspective“. In: Geopolitics, Bd. 10, Nr., S. 633-649.

Caplan, Lionel (1995): Warrior Gentlemen. ,, Gurkhas “ in the Western Imagination. Oxford.

Chadda, Maya (2002): „Integration through Internal Reorganization: Containing Ethnic Conflict in India“. In: The Global Review of Ethnopolitics, Bd. 2, Nr. 1, S. 44-61.

Chakrabarty, Dyutis (1988): Gorkhaland: Evolution of the Politics of Segregation. Special Lecture X. North Bengal University.

Chatterji, Aditi (2007): Contested Landscapes: The Story of Darjeeling. Kalkutta.

Delaney, David (2005): Territory. A short introduction. Malden, MA.

Foucault, Michel, \& Jay Miskowiec (1986): „Of Other Spaces“. In: Diacritics, Bd. 16, Nr. 1, S. 22-27.

Ghising, Subash (1987): „Letter of GNLF President Shri Subash Ghising to Sri Rajiv Gandhi, Prime Minister of India Dated 22nd July, 1987“. In: Samanta 2000, S. 271-278.

GLF - Gorkha Liberation Front (Hg.) (2009): Why Gorkhaland? Darjeeling.

Gregory, Derek (1995): „Imaginative Geographies“. In: Progress in Human Geography, Bd. 19, Nr. 4, S. 447-485.

Jones, Rhys, \& Carwyn Fowler (2007): „Placing and Scaling the Nation“. In: Environment and Planning D-Society \& Space, Bd. 25, Nr. 2, S. 332-354.

Kößler, Reinhart (2007): ,Streben nach Heimat und Freiheit. Zur Territorialisierung von Ethnizität in Süd- und Zentralnamibia“. In: PERIPHERIE, Nr. 108, S. 393-410.

Lefebvre, Henri (1991) [1974]: The Production of Space. Malden, MA.

Lossau, Julia (2002): Die Politik der Verortung. Eine postkoloniale Reise zu einer Anderen Geographie der Welt. Bielefeld.

McAdam, Doug; John D. McCarthy \& Mayer N. Zald (1996): Comparative Perspectives on Social Movements: Political Opportunities, Mobilizing Structures, and Cultural Framings. Cambridge.

MacLeod, Gordon, \& Martin Jones (2001): „Renewing the Geography of Regions“. In: Environment and Planning D-Society \& Space, Bd. 19, Nr. 6, S. 669-695.

Marston, Sallie A. (2000): „The Social Construction of Scale“. In: Progress in Human Geography, Bd. 24, Nr. 2, S. 219-242.

Massey, Dorren, \& Nigel Thrift (2003): „The Passion of Place“. In: Johnston, Ron, \& Michael Williams (Hg.): A Century of British Geography. Oxford, S. 275-302. 
Mawdsley, Emma (2002): „Redrawing the Body Politic. Federalism, Regionalism and the Creation of New States in India“. In: Commonwealth \& Comparative Politics, Bd. 40, Nr. 3, S. 34-54.

Moore, Adam (2008): „Rethinking Scale as a Geographical Category. From Analysis to Practice“. In: Progress in Human Geography, Bd. 32, Nr. 2, S. 203-225.

Newman, David (2010): „Territory, Compartments and Borders. Avoiding the Trap of the Territorial Trap“. In: Geopolitics, Bd. 15, Nr. 4, S. 773-778.

Ó Tuathail, Gearoid, \& John Agnew (1992): „Geopolitics and Discourse - Practical Geopolitical Reasoning in American Foreign-Policy“. In: Political Geography, Bd. 11, Nr. 2, S. 190-204.

Paasi, Anssi (2001): „Bounded Spaces in the Mobile World. Deconstructing 'Regional Identity"“. In: Tijdschrfit voor Economische en Sociale Geografie, Bd. 93, Nr. 2, S. 137-148.

Paasi, Anssi (2002): „Place and Region. Regional Worlds and Words“. In: Progress in Human Geography, Bd. 26, Nr. 6, S. 802-811.

Prakash, Amit (2001): Jharkhand. Politics of Development and Identity. Hyderabad.

Radcliffe, Sarah A. (1998): „Frontiers and Popular Nationhood. Geographies of Identity in the 1995 Ecuador-Peru Border Dispute“. In: Political Geography, Bd. 17, Nr. 3, S. 273-293.

Ramachandran, Sudha (2011): „Gorkhas divided over autonomy accord“. In: Asia Times, http:/www.atimes.com/atimes/South_Asia/MG21Df01.html, letzter Aufruf: 2. 11. 2011.

Reuber, Paul (1999): Raumbezogene politische Konflikte. Geographische Konfliktforschung am Beispiel von Gemeindegebietsreformen. Stuttgart.

Said, Edward (1993): Culture and Imperialism. London.

Said, Edward (1978): Orientalism. Harmontsworth.

Samanta, Amiya K. (2000): Gorkhaland Movement. A study in Ethnic Separatism. Neu-Delhi.

Schetter, Conrad, \& Markus Weissert (2007): „Die Macht des Raumes. Wahrnehmung, Legitimation und Gewalt zwischen ethnoscape und nationalem Territorium“. In: PERIPHERIE, Nr. 108, S. 376-392.

Sinha, Awesh C. (2003): „The Indians of Nepali Origin and Security of Northeast India“. In: ders. \& Tanka B. Subba (Hg.): The Nepalis in Northeast India. A Community in Search of Indian Identity. Neu-Delhi, S. 360-377.

Smith, Anthony D. (1996a): „Culture, Community, and Territory. the Politics of Ethnicity and Nationalism“. In: International Affairs, Bd. 72, Nr. 3, S. 445-458.

Smith, Anthony D. (1996b): „LSE Centennial Lecture: The Resurgence of Nationalism? Myth and Memory in the Renewal of Nations“. In: British Journal of Sociology, Bd. 47, Nr.4, S. 575-598.

Snow, David, \& Robert Benford (1988): „Ideology, Frame Resonance, and Participant Mobilization“. In: International Social Movements Research. Bd. 1, Nr. 1, S. 197-217.

Subba, Tanka B. (1992): Ethnicity, State and Development. A Case Study of the Gorkhaland Movement in Darjeeling. Neu-Delhi.

Subba, Tanka B. (2009): „The Last Word so Far...“. In: ders.; Awesh C. Sinha; G.S. Nepal \& D.R. Nepal (Hg.): Indian Nepalis. Issues and Perspectives. New Delhi, S. 383-393.

Thulung, Alok (2008): „Why Gorkhaland“. Interview with Alok Thulung in Darjeeling Times vom 23. 7. 2008, http://www.darjeelingtimes.com/news/Interviews/Why-Gorkhaland.html, letzter Aufruf: 2. 10. 2011.

Tillin, Louise (2011): „Questioning Borders: Social Movements, Political Parties and the Creation of New States in India“. In: Pacific Affairs, Bd. 84, Nr. 1, S. 67-87.

Travares, David, \& Marc Brosseau (2006): „,The Representation of Mongolia in Contemporary Travel Writing: Imaginative Geographies of a Travellers 'Frontier'“. In: Social \& Cultural Geography, Bd. 7, Nr. 2, S. 299-317.

Unified Nepal Nationalist Front (2011): Greater Nepal. http://www.greaternepal.org/, letzter Aufruf: 10. 4. 2011. 
Van Houtum, Henk; Olivier Kramsch \& Wolfgang Zierhofer (Hg.) (2005): B/ordering Space. Aldershot \& Burlington, VT.

Werlen, Benno (2005): ,Regions and Everyday Regionalizations. From a Space-Centred towards an Action-Centred Human Geography“. In: Van Houtum u.a. 2005, S. 47-60.

Werlen, Benno (1995): Sozialgeographie alltäglicher Regionalisierungen. Bd. 2: Globalisierung, Region und Regionalisierung. Stuttgart.

Anschrift der Autorin:

Miriam Bishokarma

miriam.bishokarma@uzh.ch. 